\title{
Construction of Statistical SVM based Recognition Model for Handwritten Character Recognition
}

\section{Yasir Babiker Hamdan,} International University of Africa (IUA), Khartoum, Sudan. yasir20ap@iua.edu.sd

Prof. Sathish, Department of EEE, Eritrea Institute of Technology, Eritrea. sathesh4you@gmail.com

\begin{abstract}
There are many applications of the handwritten character recognition (HCR) approach still exist. Reading postal addresses in various states contains different languages in any union government like India. Bank check amounts and signature verification is one of the important application of HCR in the automatic banking system in all developed countries. The optical character recognition of the documents is comparing with handwriting documents by a human. This OCR is used for translation purposes of characters from various types of files such as image, word document files. The main aim of this research article is to provide the solution for various handwriting recognition approaches such as touch input from the mobile screen and picture file. The recognition approaches performing with various methods that we have chosen in artificial neural networks and statistical methods so on and to address nonlinearly divisible issues. This research article consisting of various approaches to compare and recognize the handwriting characters from the image documents. Besides, the research paper is comparing statistical approach support vector machine (SVM) classifiers network method with statistical, template matching, structural pattern recognition, and graphical methods. It has proved Statistical SVM for OCR system performance that is providing a good result that is configured with machine learning approach. The recognition rate is higher than other methods mentioned in this
\end{abstract}

Information Technology

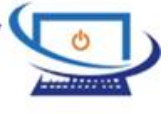


Journal of Information Technology and Digital World (2021)

Vol. 03/ No. 02

Pages: 92-107

https://www.irojournals.com/itdw/

DOI: https://doi.org/10.36548/jitdw.2021.2.003

research article. The proposed model has tested on a training section that contained various stylish letters and digits to learn with a higher accuracy level. We obtained test results of $91 \%$ of accuracy to recognize the characters from documents. Finally, we have discussed several future tasks of this research further.

Keywords: Handwriting character recognition, Support Vector Machine

\section{INTRODUCTION}

The recognition of handwritten characters is a research field in artificial intelligence, computer vision, and pattern recognition. Computers that perform handwriting recognition are said to be able to acquire and detect characters and convert them into machine-encoded form in paper papers, pictures, touch screens, and other sources. Figure 1 shows the types of the handwritten character recognition system.

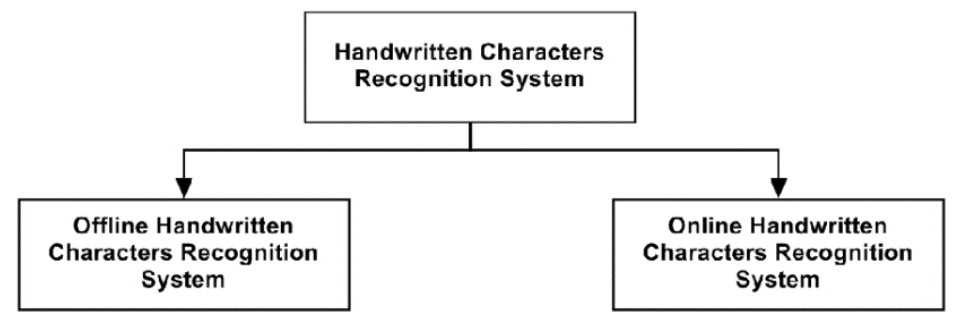

Figure 1 Type of HCR system

Its application can be found in the recognition of optical character and advanced intelligent recognition systems. Today, most of these systems implement mechanisms for machine learning such as neural networks. Machine learning is a psychological and biological branch of artificial intelligence that addresses the learning from a set of data and can be applied in it [1]. Figure 2 shows the stylish handwritten character is converting through digital characters. 


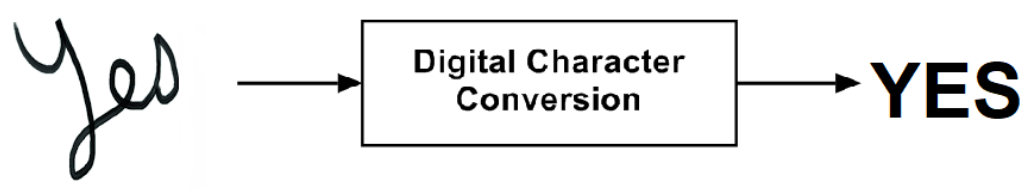

Figure 2 Basic Operation of digital conversion

K. Gaurav, Bhatia, different pre-processing technologies involve different reasonable images, from easy hand-written form-based documents with colored and complicated backgrounds with various intensities, in recognition of character and sync diagonal function extraction with offline character recognition is proposed [2]. The ANN model has been supported. For making neural network systems, like 54 functions and 69 functions, there are two approaches. Instances of data specific to a problem domain and a solution to each instance are given to a supervised machine learning model. When learning is finished, the model can provide not only answers to the data it has learned about but also highly accurate, unseen data. The researcher is concentrating on optimize techniques for text classification [3]. Machine learning models are neural networks. Their goal is to simulate the process of education in the neural system of animals or humans. Figure 3 shows the basic matrix representation of text characters.

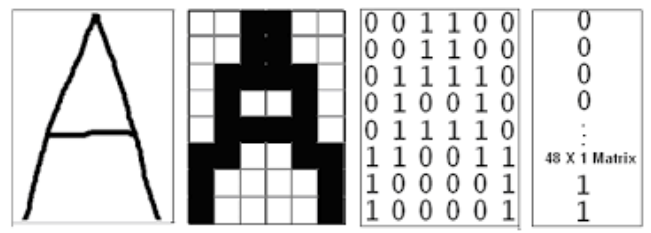

Figure 3 Basic matrix representation of text

As one of the most powerful educational models, they are useful in automating tasks where a person's choice is too long or inaccurate. In many research articles, a system for recognition of offline cursive handwriting was described, based on Hidden Markov (HMM) models by using hybrid models [4]. The figure 4 shows basic elements in neural networks such as input, hidden, and output layers.

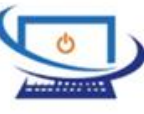




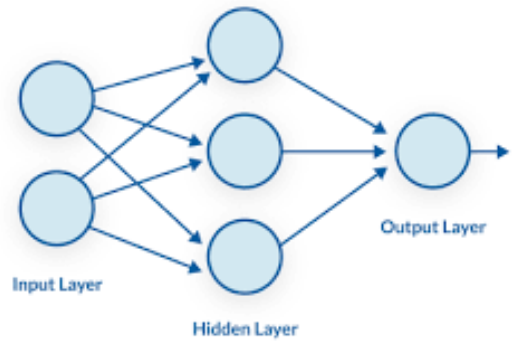

Figure 4 Basic elements of neural networks

R. Bajaj, et al employed three distinct styles of features for the classification of Devanagari numerals, such as density features, time features, and descriptive component features. For handwritten Devanagari numerals, they achieved 89.6 percent accuracy [5]. This has been carried out by many researchers using the box approach and also 90 percent [6]. A neural network can provide results very quickly and can detect links between visual instances of human data. We decided in an Android application to set up a neural network, which recognizes hand-written characters on the device touchscreen from camera and device images. The knowledge explained in this text is acquired and, without the use of libraries, the neon network has been implemented at a low level. The researcher [7]. This allows us to assess the performance of neural networks in the problem and provide network source code to solve many different classification issues. The result is a subset of a complex OCR or ICR system, which will be considered as future extensions [8].

This model works with a variety of sources of information. It is clear from past investigations that with a variety of information sources, this model succeeds, but for long phrases, it does not have a little accuracy [9]. There appear to be many proposed models that do not classify the long text data correctly. On the other hand, models incorporate CNN networks and show good results, due to their ability to handle long text information $[10,11]$.

\section{ORGANIZATION OF THE RESEARCH}

The research article structure is structured as follows; Section 3 provides current research papers on handwritten algorithms for character recognition. The proposal of a man-made

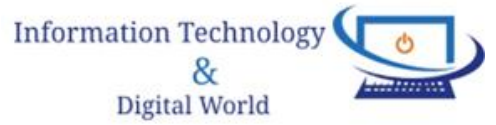


Journal of Information Technology and Digital World (2021)

Vol. 03/ No. 02

Pages: 92-107

https://www.irojournals.com/itdw/

DOI: https://doi.org/10.36548/jitdw.2021.2.003

character recognition algorithm methodology is discussed in Section 4. In section 5, the proposed algorithm and existing algorithms also describe the various examinations. Section 6 discusses the conclusion and the future task.

\section{PRELIMINARIES}

Connell and Jain [12] showed an online character recognition template system, which can represent different handwriting styles with a specific character [13]. They employed decision trees to classify characters efficiently and achieved an accuracy of $86 \%$.

The new manuscript character recognition approach for isolated Farsi-and Arabian language alphabets and digits was proposed with fractal codes by Mozaffari et al [14]. The 32 Farsi alphabets were categorized into 8 different classes based on the similarities of the characters. As a classifier for this purpose [15], a multilayer perceptron has been used. The classification rate was $87.26 \%$ and $91.37 \%$ respectively for characters and numbers.

In binary and grey pictures Liu and Suen [16] presented numeric recognition results for handwritten Bangla and Farši images. In the three public datasets of ISI Bangla numerals, IFHCDB Farsi numerals, and IFHCDB Farsi numerals, the researchers applied different character recognition methods and classifiers [17] to claim that the highest accuracy of these data sets was obtained, namely 99.40 percent, 99.16 percent, and 99.73 percent, respectively.

Researchers have been working on different approaches to machine learning in the present decade, such as Support Vector Machine, Random Forests (RF), k Nearest Neighbor $(\mathrm{kNN})$, Decision Tree (DT). These machine learning techniques have been combined with imaging methods to increase the accuracy of the recognition system. Researchers recently focused on developing digitalization techniques for handwritten documents, mainly based on a deep knowledge approach [18, 19]. This paradigm shift has been generated because cluster computers and GPUs have been adapted, and deeper learning architectures [20] are improving their performance, which includes recurring neural networks (RNN), CNNs, long-term memory (LSTM) networks, etc. The objective of this Systemic Literature Review (SLR) is not just to introduce literature in the field of OCR. 
Journal of Information Technology and Digital World (2021)

Vol. 03/ No. 02

Pages: 92-107

https://www.irojournals.com/itdw/

DOI: https://doi.org/10.36548/jitdw.2021.2.003

In different languages, but also by pointing out weak areas of the existing OCR systems requiring further investigation for new researcher research directions. The first researcher to utilize a neural network approach to identifying Indian documents was Patil and Subbareddy [21]. The researchers are suggesting a system that reads scripts in English, Hindi, and Kannada. For the identification of scripts, a modular neural network was used while a two-stage feature extraction system was developed, first to extend the image of the document to the average pixel distribution.

Sharma et al proposed a scheme for the recognition of the Devanagari script that was based on quadratic classification [22]. For feature recognition, the investigators used 64 directions based on the histogram chain code [23]. In recognition of the Devanagari characteristics, the proposed system resulted from inaccuracy of 80.36 percent and 98.86 percent. The results were calculated using five-fold cross-validation. The Devanagari text line segments method was proposed by Kumar et al [24]. A precision of $91.5 \%$ has been achieved in line segmentation and $98.1 \%$ in word segmentation. Power and Chaturvedi [25] have used the handwritten backpropagation network for recognition.

The results showed the highest rate of recognition at 98.5\%. Handwritten Numeral Script Identification or HSSI framework based on four scripts indicated by hand [26] Obaidullah et al. [27] proposed Bangla, Devanagari, Roman, and Urdu. The researchers used various classifications such as NBTree, PART, Random Forest, SMO, Simple Logistic, and MLP and assessed the performance against the real positive. It was found that MLP performance was better than the rest. MLP was then used to identify bi and tri-script. The bi-script Bangla and Urdu combination showed the highest precision rate in MLP of 90.9 percent, while a tri-script combination of languages achieved the highest accuracy rate of 74 percent for Bangla and Urdu character recognition.

\section{METHODOLOGIES}

OCR based algorithm is used for training and classifies the process to learn a framework model through given input data. In this section, we have focused on the relevant techniques and methods for OCR for HCR. 
Journal of Information Technology and Digital World (2021)

Vol. 03/ No. 02

Pages: 92-107

https://www.irojournals.com/itdw/

DOI: https://doi.org/10.36548/jitdw.2021.2.003

\subsection{Proposed statistical Based SVM Framework}

Generally, ANN consisting of several processing units connected through neurons which are connected with whole units in the system. This process is consisting of input data and a map for the predefined label. These nodes are associated and adjusted to a possible error on learning time samples in a controlled environment. Figure 5 shows the proposed framework workflow.

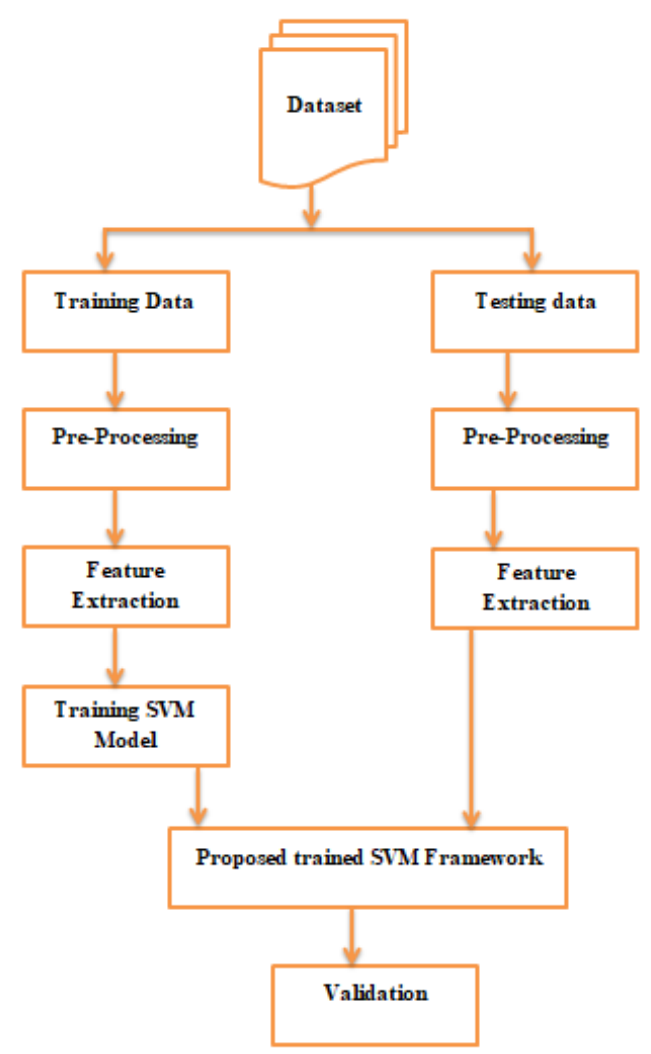

Figure 5 Classification process of proposed SVM framework

\subsubsection{Multi-layer perceptron}

The symbolic depictions of Multilayer perceptron contain three layers which are showing in figure 4. This type of network is called feed forward networks which can be replicated in the human memory to calculate by neurons. Figure 6 shows the internal architecture of statistical SVM architecture for HCR. 


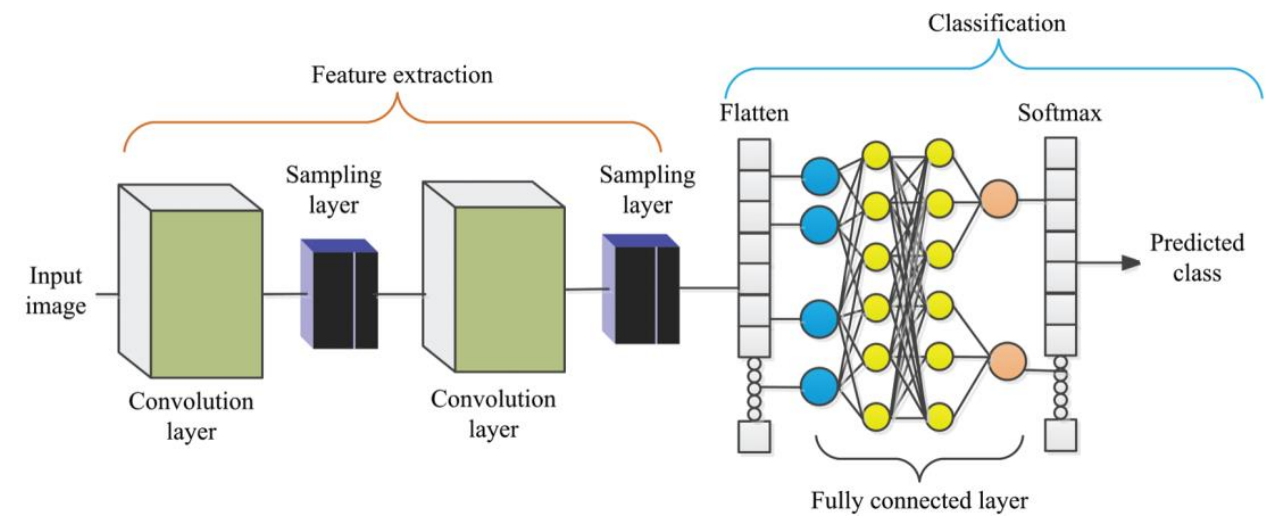

Figure 6 Proposed internal architecture for HCR

\subsubsection{Kernel Approach}

The support vector machine concepts are based on kernel-based learning models which contain principal component analysis for feature extraction. This context feature extraction can be used in optical pattern text categorization with time series prediction. This significant relevant mapping of feature vectors is performing into large dimensional space with the hyperplane that is used to separate and classifies the classes. Also, this technique proves more robust for HCR with image classification through text content in the images. Let us define,

$$
f(x)=\operatorname{sgn}\left(\sum_{k=1}^{1} s_{k} \cdot y_{k} K\left(x_{k}, x\right)+b\right)
$$

Where $\left\{\left(x_{k}, y_{k}\right), k=1 \ldots l\right\}$

The $\mathrm{K}$ is a kernel function with a threshold parameter of the hyperplane. The Lagrange multipliers of the optimization issues denoted by $s_{k}$. The figure 5 shows the proposed internal architecture for HCR. In the input of the structure is taken some sample of handwriting characters and its performed by pre-processing to register the samples.

\subsection{Statistical Method}

This method is classified into two types as parametric and non-parametric. The fixed and finite number of parameters as input data called the parametric type method. This type is usually faster learning capability with the least number of the dataset. The logical regression, hidden

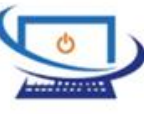


Journal of Information Technology and Digital World (2021)

Vol. 03/ No. 02

Pages: 92-107

https://www.irojournals.com/itdw/

DOI: https://doi.org/10.36548/jitdw.2021.2.003

Markov model are an example of parametric type classifiers. But the non-parametric classifiers are more flexible and more input data can be analyzed. Decision Tree, K Nearest Neighbor classifiers are examples of non-parametric methods that can have the capability to learn with a large number of the dataset. Here we are taking non-parametric methods to analyze and compare with our proposed framework. Usually, the kNN classifier is used as a non-parametric statistical method in OCR. The kNN is finding the closest target function in the training samples with the statistical approach. The probability of output for an unknown sample is defined as,

$$
P\left(\frac{y}{S}\right)=\frac{\sum_{k \in K} W_{k} \cdot 1_{\left(k_{y}=y\right)}}{\sum_{k \in K} W_{k}}
$$

Where $\mathrm{k}$ is the nearest neighbors, $\mathrm{k}_{\mathrm{y}}$ is the output class.

The character recognition achieves better accuracy in the examination with various datasets. kNN classifiers assign a class closest neighbors in the weight vectors inside the boundary.

\subsection{Template Matching Method (TMM)}

This type of method is consisting of many templates that contain the information which will compare with unknown samples. Generally, the matching templates choose the closest matching features. Besides predefined templates are employing as a sliding window approach to compare between original in the dataset with unknown input samples. After the characters are matching with predefined templates, the classification can be happening. The similarity metrics are used in this method to measure the similarities with Euclidean distance with correlation function [28].

\subsection{Structural pattern acknowledgement (SPA)}

This method is an ancient method to recognize the pattern through the structural network which is shown in the figure. This pattern aims to recognize the objects through various structural points' collections for various objects by edges and boundaries components. This method is used for OCR through chain code histogram techniques. The binary values of boundaries are well defined to recognize characters and classify them through the chain code histogram technique [29]. Figure 7 shows the basic structural pattern acknowledgment. 


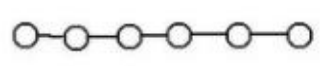

String

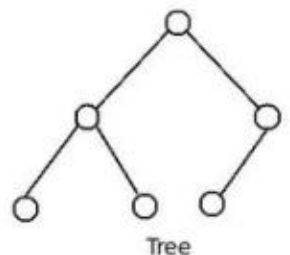

Tree

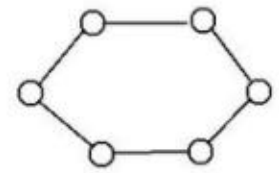

Graph

Figure 7 basic structural pattern acknowledgements

\section{RESULTS DISCUSSION}

This research article is comparing various OCR algorithms based on standardized datasets for better evaluation.

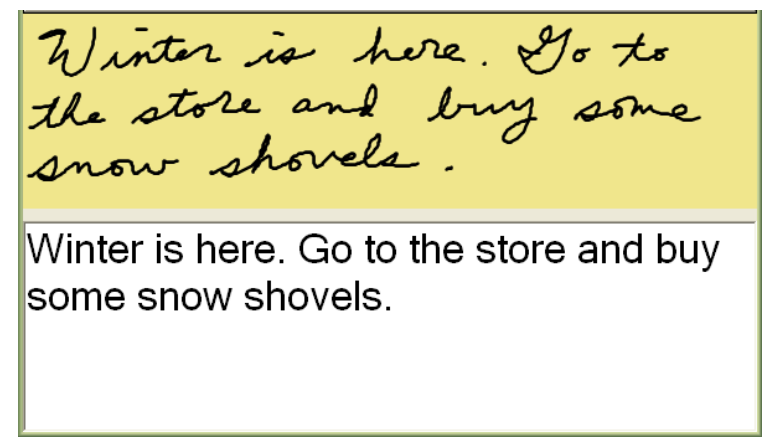

Figure 8 Output results of stylish text characters recognition after examination

The fundamental requirement of testing any proposed model with existing methods should have enough amounts of data for training purposes. Here $80 \%$ and $20 \%$ of data are used from the dataset for training and testing purposes respectively.

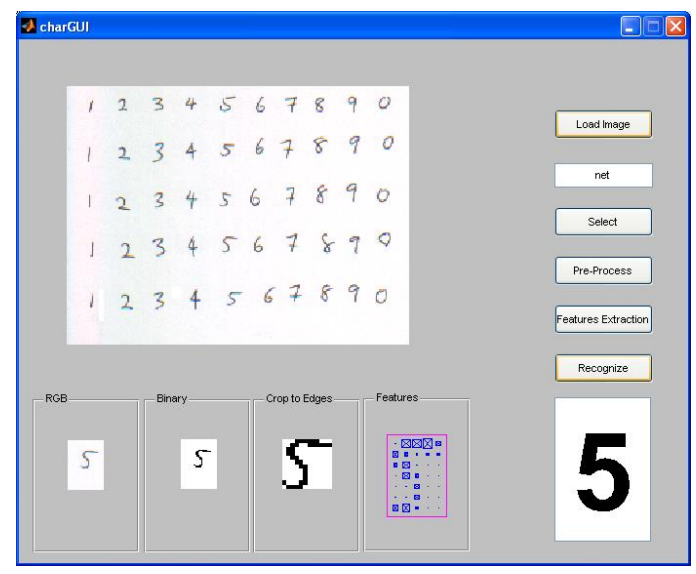

Figure 9 Output results of stylish numeral characters recognition after examination 
Journal of Information Technology and Digital World (2021)

Vol. 03/ No. 02

Pages: 92-107

https://www.irojournals.com/itdw/

DOI: https://doi.org/10.36548/jitdw.2021.2.003

We have used various languages in the testing for character recognition such as MNIST, CENPARMI, UCOM, and IAM. This HCL2000 datasets is containing Chinese characters. The mentioned algorithm does not contain any italic stylish text characters except our proposed algorithm. Figure 8 and 9 shows the obtained output results of stylish text and numeral characters recognition after test respectively.

Table 1 Overall performance measures of proposed framework

\begin{tabular}{|c|c|c|c|c|c|c|}
\hline S.No & Methods & Accuracy & $\begin{array}{c}\text { Word } \\
\text { Recognition } \\
\text { Rate }\end{array}$ & Sensitivity & Efficiency & $\begin{array}{c}\text { Computation } \\
\text { Time }\end{array}$ \\
\hline 1 & Statistical Method & $86 \%$ & $86.37 \%$ & $82 \%$ & $82 \%$ & Low \\
\hline 2 & $\begin{array}{c}\text { Template Matching } \\
\text { Method }\end{array}$ & $65 \%$ & $75.2 \%$ & $71 \%$ & $71 \%$ & Medium \\
\hline 3 & $\begin{array}{c}\text { Structural Pattern } \\
\text { Acknowledgement }\end{array}$ & $75 \%$ & 80.2 & $70 \%$ & $70 \%$ & High \\
\hline 4 & $\begin{array}{c}\text { Proposed } \\
\text { Statistical SVM }\end{array}$ & $88 \%$ & $91.1 \%$ & $89 \%$ & $91 \%$ & High \\
\hline
\end{tabular}

One of the widely using languages in the world is English. Many countries are having English as official language. Our examination, we used English letter reconstruction from English handwritten characters. This recognition has been carried out here for OCR algorithm.

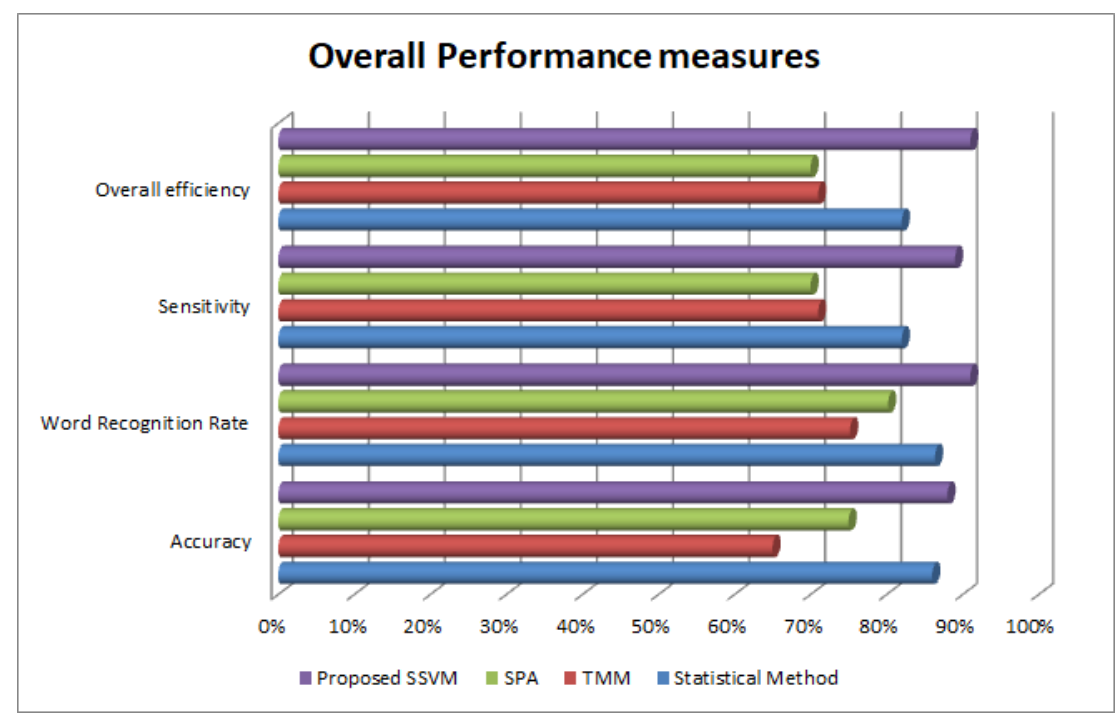

Figure 10 shows the graph of overall performance evaluation between various OCR algorithms. 
Journal of Information Technology and Digital World (2021)

Vol. 03/ No. 02

Pages: 92-107

https://www.irojournals.com/itdw/

DOI: https://doi.org/10.36548/jitdw.2021.2.003

Here we have used many commercial applications' dataset to conduct test the OCR system for English language successfully. The state of art in the field of character recognition is conducted automatically. This result is achieved after segmentation of electronic ink in the paper. The numeral character recognition test conducted with our statistical SVM method and got good accuracy even though stylish numeral text which is shown in the figure 9. Table 1 shows the results calculated from the measuring metrics for evaluate the proposed framework.

\section{CONCLUSION}

Thus our proposed approach proves the superiority to recognize the character with higher accuracy effectively among all types of OCR. This research article developed the framework to recognize various stylish characters inclusion provides better recognition rate and accuracy. Most of the research works are fail to incorporate enough dataset in their examination. Our proposed framework succeeds to achieve better accuracy with the inclusion of a stylish character recognition procedure. The writing style can depend on various handwriting characters with distorted strokes and variable thickness of italic characters. Our proposed SVM-based HCR method gives $94 \%$ accuracy and a good recognition rate while compared to existing methods. The input image was preprocessed and extracted features for getting higher accuracy. We used enough amount of dataset to recognize the handwriting characters in real-time scenarios and pictorial illumination as well. Our research work can be further extended with various possibilities as follows;

1) Research in the OCR domain in some of the most commonly spoken languages is usually conducted here. This is partly because the data sets in other languages are not available. One way of future research involves researching different languages, i.e. regional languages and endangered languages, other than widely spoken languages. This can contribute to the preservation of the cultural heritage of vulnerable communities and the development of global synergy.

2) The research community needs to develop systems for recognizing text and characters on-screen on various conditions in everyday scenarios, e.g. text in roadway signboards and billboards. This is the "recognition/classification/text domain in the wild" domain. This is a complex problem as a system for which background conflicts, variable lighting conditions, 
Journal of Information Technology and Digital World (2021)

Vol. 03/ No. 02

Pages: 92-107

https://www.irojournals.com/itdw/

DOI: https://doi.org/10.36548/jitdw.2021.2.003

variable camera angles, distorted characteristics, and variable writing styles need to be addressed [30].

3) Researchers need to develop tough and comprehensive datasets to incorporate all potential variations in characters to build a sturdy system for "text in the wild." Characters written by various persons create large variability intra-class, making it difficult for classifiers to work robustly. Although computational complications (specifically during the classifier training phase) have been increased with the increasing use of complex profound learning architectures, classification exactness has improved. This creates a barrier to developing a robust hand recognition system in real-time.

4) Published studies have proposed different OCR systems, but the marketing of research should be an improvement. Marketing Research will contribute to the development of low-cost real-life OCR systems which can turn many invaluable data into searching context [31].

\section{REFERENCES}

[1] X. Feng, H. Yao, and S. Zhang, "Focal CTC loss for Chinese optical character recognition on unbalanced datasets" Complexity, vol. 2019, Jan. 2019, Art. no. 9345861.

[2] L. Xu, Y. Wang, X. Li, and M. Pan, "Recognition of handwritten Chinese characters based on concept learning,'” IEEE Access, vol. 7, pp. 102039-102053, 2019.

[3] Manoharan, J. Samuel. "Capsule Network Algorithm for Performance Optimization of Text Classification" Journal of Soft Computing Paradigm (JSCP) 3, no. 01 (2021): 1-9.

[4] M. Ahmed and A. I. Abidi, "Performance comparison of ANN and template matching on English character recognition,' Int. J. Advance Res., Ideas Innov. Technol., vol. 5, no. 4, pp. 367-372, 2019.

[5] Chakrabarty, Navoneel, and Sanket Biswas. "Navo Minority Over-sampling Technique (NMOTe): A Consistent Performance Booster on Imbalanced Datasets." Journal of Electronics 2, no. 02 (2020): 96-136.

Information Technology \& Digital World 
Journal of Information Technology and Digital World (2021)

Vol. 03/ No. 02

Pages: 92-107

https://www.irojournals.com/itdw/

DOI: https://doi.org/10.36548/jitdw.2021.2.003

[6] M. Yashodha, S. Niranjan, and V. N. M. Aradhya, "Deep learning for trilingual character recognition' Int. J. Natural Comput. Res., vol. 8, no. 1, pp. 52-58, Jan. 2019.

[7] Adam, Edriss Eisa Babikir. "Evaluation of Fingerprint Liveness Detection by Machine Learning Approach-A Systematic View" Journal of ISMAC 3, no. 01 (2021): 16-30. DOI: 10.36548/jismac.2021.1.002.

[8] A. Yousaf, M. J. Khan, M. J. Khan, N. Javed, H. Ibrahim, K. Khurshid, and K. Khurshid, "Size invariant handwritten character recognition using single layer feedforward backpropagation neural networks," in Proc. 2nd Int. Conf. Comput., Math. Eng. Technol. (iCoMET), Jan. 2019, pp. 1-7.

[9] Adam, Edriss Eisa Babikir. "Deep Learning based NLP Techniques In Text to Speech Synthesis for Communication Recognition." Journal of Soft Computing Paradigm (JSCP) 2, no. 04 (2020): 209-215.

[10] S. Karthik and K. S. Murthy, "Deep belief network based approach to recognize handwritten Kannada characters using distributed average of gradients," Cluster Comput., vol. 22, no. S2, pp. 4673-4681, Mar. 2019.

[11] Hamdan, Yasir Babiker. "Faultless Decision Making for False Information in Online: A Systematic Approach." Journal of Soft Computing Paradigm (JSCP) 2, no. 04 (2020): 226-235. DOI: https://doi.org/10.36548/jscp.2020.4.004.

[12] S. D. Connell and A. K. Jain, "Template-based online character recognition," Pattern Recognit., vol. 34, no. 1, pp. 1_14, Jan. 2001.

[13] Manoharan, Samuel, and Narain Ponraj. "Analysis of Complex Non-Linear Environment Exploration in Speech Recognition by Hybrid Learning Technique." Journal of Innovative Image Processing (JIIP) 2, no. 04 (2020): 202-209.

[14] S. Mozaffari, K. Faez, and H. R. Kanan, "Recognition of isolated handwritten Farsi/Arabic alphanumeric using fractal codes," in Proc. 6th IEEE Southwest Symp. Image Anal. Interpretation, Mar. 2004, pp. 104_108.

Information Technology \& Digital World 
Journal of Information Technology and Digital World (2021)

Vol. 03/ No. 02

Pages: 92-107

https://www.irojournals.com/itdw/

DOI: https://doi.org/10.36548/jitdw.2021.2.003

[15] Haoxiang, Wang, and S. Smys. "Overview of Configuring Adaptive Activation Functions for Deep Neural Networks-A Comparative Study." Journal of Ubiquitous Computing and Communication Technologies (UCCT) 3, no. 01 (2021): 10-22.

[16] C.-L. Liu and C. Y. Suen, "A new benchmark on the recognition of handwritten bangla and farsi numeral characters," Pattern Recognit., vol. 42, no. 12, pp. 3287_3295, Dec. 2009.

[17] Karuppusamy, P. "Building Detection using Two-Layered Novel Convolutional Neural Networks." Journal of Soft Computing Paradigm (JSCP) 3, no. 01 (2021): 29-37.

[18] Y. LeCun, Y. Bengio, and G. Hinton, "Deep learning" Nature, vol. 521, no. 7553, pp. 436_444, 2015.

[19] Duraipandian, M. "Adaptive Algorithms for Signature Wavelet recognition in the Musical Sounds." Journal of Soft Computing Paradigm (JSCP) 2, no. 02 (2020): 120-129.

[20] T. M. Breuel, A. Ul-Hasan, M. A. Al-Azawi, and F. Shafait, "High performance OCR for printed English and Fraktur using LSTM networks," in Proc. 12th Int. Conf. Document Anal. Recognit., Aug. 2013, pp. 683_687.

[21] S. B. Patil and N. V. Subbareddy, "Neural network based system for script identification in Indian documents," Sadhana, vol. 27, no. 1, pp. 83_97, Feb. 2002.

[22] N. Sharma, U. Pal, F. Kimura, and S. Pal, "Recognition of off-line handwritten devnagari characters using quadratic classifier" in Computer Vision, Graphics and Image Processing. Berlin, Germany: Springer, 2006, pp. 805_816.

[23] H. Freeman and L. Davis, "A corner finding algorithm for chain coded curves," IEEE Trans. Comput., vol. C-26, no. 3, pp. 297_303, Mar. 1977.

[24] N. K. Garg, D. L. Kaur, and D. M. Kumar, "Segmentation of handwritten hindi text," Int. J. Comput. Appl., vol. 1, no. 4, pp. 22_26, Feb. 2010

[25] Y. Perwej and A. Chaturvedi, "Machine recognition of hand written characters using neural networks," 2012, arXiv:1205.3964. Available: http://arxiv.org/abs/1205.3964.

[26] S. M. Obaidullah, C. Halder, N. Das, and K. Roy, "Numeral script identification from handwritten document images," Procedia Comput.Sci., vol. 54, pp. 585_594, Jan. 2015.

[27] S. Mozaffari, K. Faez, and H. R. Kanan, "Recognition of isolated handwritten Farsi/Arabic alphanumeric using fractal codes," in Proc. 6th IEEE Southwest Symp. Image Anal. Interpretation, Mar. 2004, pp. 104_108.

Information Technology \& Digital World 
Journal of Information Technology and Digital World (2021)

Vol. 03/ No. 02

Pages: 92-107

https://www.irojournals.com/itdw/

DOI: https://doi.org/10.36548/jitdw.2021.2.003

[28] S. Kowsalya and P. Periasamy, "Recognition of tamil handwritten character using modified neural network with aid of elephant herding optimization," Multimedia Tools Appl., vol. 78, no. 17, pp. 25043-25061, 2019.

[29] S. B. Ahmed, S. Naz, S. Swati, and M. I. Razzak, "Handwritten Urdu character recognition using one-dimensional BLSTM classifier," Neural Comput. Appl., vol. 31, no. 4, pp. 11431151, Apr. 2019.

[30] Duraipandian, M. "Ranked k-NN Crowd sourced Model for Cloud Internet of Things (CIoT)." Journal of ISMAC 2, no. 03 (2020): 173-180.

[31] M. J. Rafeeq, Z. U. Rehman, A. Khan, I. A. Khan, and W. Jadoon, "Ligature categorization based nastaliq Urdu recognition using deep neural networks," Comput. Math. Org. Theory, vol. 25, no. 2, pp. 184-195, Jun. 2019. 